\title{
Overlapping Computation/Communication in the Parallel One-Sided Jacobi Method ${ }^{\star}$
}

\author{
El Mostafa Daoudi, Abdelhak Lakhouaja, and Halima Outada \\ University of Mohammed First, Faculty of Sciences \\ Department of Mathematics and Computer Science \\ LaRI Laboratory, 60000 Oujda, Morocco \\ e-mail : \{mdaoudi,lakhouaja,outada\}@sciences.univ-oujda.ac.ma
}

\begin{abstract}
In this work, we propose some techniques for overlapping the communication by the computation in the parallelization of the one-sided Jacobi method for computing the eigenvalues and the eigenvectors of a real and symmetric matrix. The proposed techniques are experimented on a cluster of PCs and on the parallel system TN310.
\end{abstract}

\section{Introduction}

In this paper, we study, on distributed memory architecture, the parallelization of the one-sided Jacobi method for computing the eigenvalues and the eigenvectors of a real symmetric matrix $A$ of size $n$. The one-sided algorithm is better suited to parallel computers [26], since it needs less communications than the classical Jacobi method ("two-sided Jacobi method" [3]). It only needs one-to-one communications type (column translation) in contrast to the two-sided Jacobi method which needs, in addition, global communications (rotations broadcast) [8|4. On the other hand, the computation time complexity of the one-sided and the two sided (without exploiting the symmetry) algorithms are equivalent for computing the eigenvalues and the eigenvectors. Our objective in this study is to propose some techniques for overlapping the communications by the computations during the translation phase by carrying out simultaneously the update and the translation of one column 5 .

We consider a distributed memory architecture composed of $p$ processors, denoted by $P_{m}, 0 \leq m \leq p-1$. The communication cost of $L$ data between two neighbor processors is modeled by $\operatorname{comm}(L)$ and the computation time of one floating point operation is designed by $\omega$.

\section{Sequential Algorithm}

The basic idea of the Jacobi method [3] consists in constructing the sequences of matrices $\left\{A^{(k+1)}=J^{(k)^{\top}} A^{(k)} J^{(k)}\right\}$ and $\left\{U^{(k+1)}=U^{(k)} J^{(k)}\right\}$ which converge respectively to a diagonal matrix that contains the eigenvalues and

* Supported by the "Comité mixte Franco-Marocain", AI $n^{o}:$ MA 01/19, and by the European INCO-DC Program, "DAPPI" Project 
to a matrix that contains the eigenvectors. Where $A^{(1)}=A, U^{(1)}=I_{n}$ and $J^{(k)}=J(i, j)$, for $1 \leq j<i \leq n$, is a Jacobi rotation, in the $(i, j)$ plane, chosen in order to annihilate $a_{i, j}^{(k)}=a_{j, i}^{(\bar{k})}$. It is completely determined by $a_{i, i}^{(k)}, a_{j, j}^{(k)}$ and $a_{i, j}^{(k)}$.

The one-sided Jacobi method: The application of the rotation $J(i, j)$ to the matrix, requires to update the columns $i$ and $j$ of the matrix and by symmetry the rows $i$ and $j$. This update needs to exchange the rotations, which leads to an all-to-all communication type (global synchronization). In order to avoid the global synchronizations, one constructs the sequence $\left\{\bar{A}^{(k+1)}=\bar{A}^{(k)} J^{(k)}\right\}$ instead of the sequence $\left\{A^{(k)}\right\}$, where $\bar{A}^{(1)}=A$. At each step $k$ of the algorithm, one computes one rotation (determination of $J^{(k)}$ ) and one updates two columns of $\bar{A}^{(k)}$ and two columns of $U^{(k)}$. Since the determination of $J^{(k)}=J(i, j)$, needs the knowledge of the coefficients $a_{i, i}^{(k)}, a_{j, j}^{(k)}$ and $a_{i, j}^{(k)}$, and since, we only know $\bar{A}^{(k)}=\left(\bar{A}_{1}^{(k)}, \cdots, \bar{A}_{n}^{(k)}\right)$ and $U^{(k)}=\left(U_{1}^{(k)}, \cdots, U_{n}^{(k)}\right)$, these coefficients can be computed by using the following relation [6]: $a_{m, l}^{(k)}=\left(U_{m}^{(k)}\right)^{\top} \bar{A}_{l}^{(k)}$ for all $1 \leq$ $m, l \leq n$ which requires $6 n \omega$.

\section{Study of the Parallelization of the One-Sided Jacobi}

We consider a column distribution of $\bar{A}$ and $U$. Each processor $P_{m}$, for $0 \leq m \leq$ $p-1$, holds $\frac{n}{p}$ columns of $\bar{A}$, denoted by $\bar{A}_{m_{1}(j)}, \bar{A}_{m_{2}(j)}$ and $\frac{n}{p}$ columns of $\bar{U}$ denoted by $U_{m_{1}(j)}$ and $U_{m_{2}(j)}$, for $1 \leq j \leq \frac{n}{2 p}$. Initially, we have $m_{1}(i)=m \frac{n}{p}+i$, for $1 \leq i \leq \frac{n}{2 p}$ and $m_{2}(i)=m \frac{n}{p}+i$, for $\frac{n}{2 p}+1 \leq i \leq \frac{n}{p}$, which corresponds to $\frac{n}{p}$ consecutive columns of $A$ and $\frac{n}{p}$ consecutive columns of $U$.

The elements are annihilated according to the block odd-even ordering presented in 6]. During each step, all possible rotations are performed as shown on figure 1 for $n=12$ and $p=3$. For example, at the first step, $P_{1}$ holds the columns $5,6,7$ and 8 , then it performs all possible rotations $(5,6),(5,7),(5,8),(6,7)$, $(6,8)$ and $(7,8)$. At the second step, it holds the columns $1,2,7$ and 8 , then it performs all possible rotations $(1,7),(1,8),(2,7)$ and $(2,8)$. The rotations $(1,2)$ and $(7,8)$ are done during the first step.
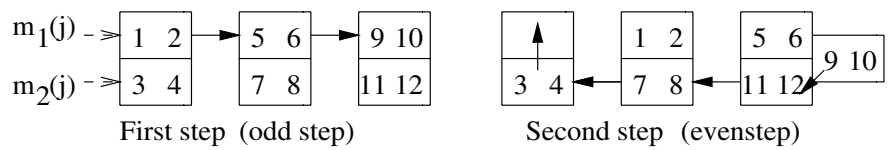

Fig. 1. The first and second steps of the block ordering for $n=12$ and $p=3$. The arrows show the sense of the column translation.

There are two phases for the parallelization. At each step, each processor:

- Phase 1: computes all possible local rotations and updates its local columns: 
- in the first step, it computes all possible rotations $J\left(m_{1}(i), m_{2}(j)\right)$, $J\left(m_{1}(i), m_{1}(j)\right)$ and $J\left(m_{2}(i), m_{2}(j)\right)$ for $1 \leq i<j \leq \frac{n}{2 p}$, which corresponds to $\frac{\frac{n}{p}\left(\frac{n}{p}-1\right)}{2}$ rotations.

- during the other steps, for $1 \leq i, j \leq \frac{n}{2 p}$, it computes the rotations $J\left(m_{1}(i), m_{2}(j)\right)$, which corresponds to $\left(\frac{n}{2 p}\right)^{2}$ rotations.

- Phase 2: communicates only with its two neighbors in order to send and receive $\frac{n}{2 p}$ columns of $\bar{A}$ and $\frac{n}{2 p}$ columns of $U$, in order to complete a sweep.

\section{Overlapping the Communications}

The basic idea to overlap the communication with the computation is to delay the computation of some rotations in order to be computed simultaneously during the translation phase. We assume that at one given step, the processor $P_{m}$, for $0 \leq m \leq p-1$, holds the columns $\bar{A}_{m_{1}(j)}, \bar{A}_{m_{2}(j)}, U_{m_{1}(j)}$ and $U_{m_{2}(j)}$, for $1 \leq j \leq \frac{n}{2 p}$ :

- it computes all possible rotations (illustrated by black squares in the figures 2) except the rotations $J\left(m_{1}(j), m_{2}(j)\right)$, for $1 \leq j \leq \frac{n}{2 p}$ (rotations illustrated by white squares in the figures 2).

- it updates the corresponding columns.

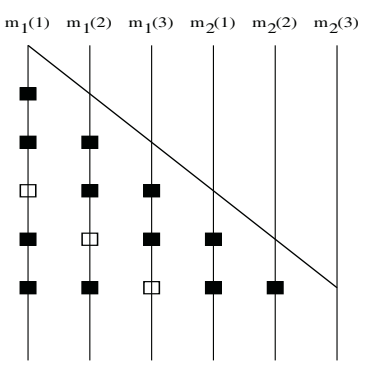

(a) first step

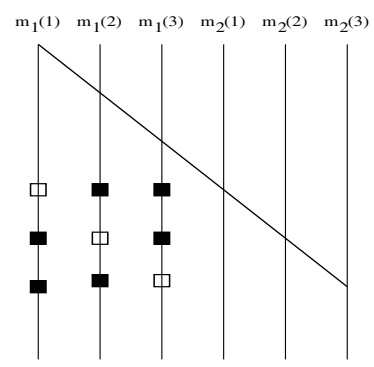

(b) other steps

Fig. 2. Computation of the rotations during the overlapping phase.

The translation will be performed simultaneously with the computation of the remaining rotations $J\left(m_{1}(j), m_{2}(j)\right)$, for $1 \leq j \leq \frac{n}{2 p}$.

\subsection{First Strategy}

The theoretical study of this strategy is developed in [5]. Each processor computes $J\left(m_{1}(j), m_{2}(j)\right)$ and updates $\bar{A}_{m_{2}(j)}$ and $U_{m_{2}(j)}$, then, simultaneously: 
- updates $\bar{A}_{m_{1}(j)}$ and $U_{m_{1}(j)}$

- sends $\bar{A}_{m_{2}(j)}$ and $U_{m_{2}(j)}$ according to the odd-even ordering.

The update of one column needs $3 n \omega$ and the computation of one rotation needs $6 n \omega$, while the communication of two columns needs $\operatorname{comm}(2 n)$

Lemma: If $6 n \omega \geq \operatorname{comm}(2 n)$, all the communications can be overlapped by the computations.

\subsection{Second Strategy}

Before translating columns, we divide each column of $\bar{A}$ and $U$ into $q$ blocks each of size $\frac{n}{q}$. The translation of each column is done in pipeline, as follow: Each processor:

- computes $J\left(m_{1}(1), m_{2}(1)\right)$ and updates the first block of the columns $\bar{A}_{m_{1}(1)}$, $U_{m_{1}(1)}, \bar{A}_{m_{2}(1)}$ and $U_{m_{2}(1)}$.

- For $1 \leq j \leq \frac{n}{2 p}-1$ :

* for $2 \leq i \leq i$, simultaneously:

- updates the $i^{t h}$ blocks of $\bar{A}_{m_{1}(j)}, U_{m_{1}(j)}, \bar{A}_{m_{2}(j)}$ and $U_{m_{2}(j)}$.

- sends the $(i-1)^{\text {th }}$ blocks of $\bar{A}_{m_{2}(j)}$ and $U_{m_{2}(j)}$.

* simultaneously:

- computes $J\left(m_{1}(j+1), m_{2}(j+1)\right)$ and updates the first block of the columns $\bar{A}_{m_{1}(j+1)}, U_{m_{1}(j+1)}, \bar{A}_{m_{2}(j+1)}$ and $U_{m_{2}(j+1)}$.

- for $j=\frac{n}{2 p}$

- sends the last blocks of $\bar{A}_{m_{2}(j)}$ and $U_{m_{2}(j)}$.

* for $2 \leq i \leq q$, simultaneously:

- updates the $i^{t h}$ blocks of $\bar{A}_{m_{1}(j)}, U_{m_{1}(j)}, \bar{A}_{m_{2}(j)}$ and $U_{m_{2}(j)}$.

- sends the $(i-1)^{\text {th }}$ blocks of $\bar{A}_{m_{2}(j)}$ and $U_{m_{2}(j)}$.

* sends the last blocks of $\bar{A}_{m_{2}(j)}$ and $U_{m_{2}(j)}$.

The update of one block of a column needs $3 \frac{n}{q} \omega$, while the communication of two blocks needs $\operatorname{comm}\left(2 \frac{n}{q}\right)$. If we assume that the communication cost of $L$ data between two neighbor processors is modeled by $\operatorname{comm}(L)=\beta+L \tau$, where $\beta$ is the start-up time and $\tau$ is the time to transmit one data, then the parallel time which necessitates this part of the parallel algorithm is modeled by:

$$
\begin{aligned}
T(q)= & 6 n \omega+\frac{12 n \omega}{q}+ \\
& \left(\frac{n}{2 p}-1\right)\left[(q-1) \max \left(\beta+\frac{2 n \tau}{q}, \frac{12 n \omega}{q}\right)+\max \left(\beta+\frac{2 n \tau}{q}, 6 n \omega+\frac{12 n \omega}{q}\right)\right]+ \\
& (q-1) \max \left(\beta+\frac{2 n \tau}{q}, \frac{12 n \omega}{q}\right)+\left(\beta+\frac{2 n \tau}{q}\right)
\end{aligned}
$$

Lemma: The optimal size which minimize the parallel time is given by:

1. $q^{*}=\min \left(\left[\frac{2 n(6 \omega-\tau)}{\beta}\right], n\right)$ if $\beta+\frac{2 n \tau}{q} \leq \frac{12 n \omega}{q}$

2. $q^{*}=\min \left(\left[2 \sqrt{\frac{6 p \omega}{\beta}}\right], n\right)$ if $6 n \omega+\frac{12 n \omega}{q} \leq \beta+\frac{2 n \tau}{q}$

3. if $\frac{12 n \omega}{q} \leq \beta+\frac{2 n \tau}{q} \leq 6 n \omega+\frac{12 n \omega}{q}$ :

a) $q^{*}=\min \left(\left[\sqrt{\frac{12 n \omega+4 p \tau-2 n \tau}{\beta}}\right], n\right)$ if $n \tau \leq 6 n \omega+2 p \tau$

b) $q^{*}=\min \left(\left[2 n \frac{6 \omega-\tau}{\beta-6 n \omega}\right], n\right)$ if $\beta-6 n \omega \geq 0$ and $6 \omega-\tau \geq 0$

The proof of this lemma is straightforward by derivating the function $T(q)$. 


\section{Experimental Results}

The proposed techniques have been implemented on our local parallel machine TN310 based on Transputers and on the cluster of PCs installed at IMAGGrenoble-France 1]. Our objective is not to compare the obtained performances on the two different parallel systems, but to compare the performances obtained of the proposed techniques on each system. For the numerical tests, we have used the Frank matrix defined by: $A=\left(a_{i, j}\right)$, where $a_{i j}=n-\max (i, j)+1$, for $i, j=1,2, \ldots, n$, which the eigenvalues are given by: $\lambda_{k}=\frac{1}{2\left(1-\cos \left(\frac{2 k-1}{2 n+1} \pi\right)\right)}$ for $k=1,2, \ldots, n$.

In table 1 (resp. table 2), we compare the execution times for one sweep on the cluster (resp. TN310) without overlapping (n_ov) and the first strategie of the overlapping (str1). The obtained results on the cluster show that the use of the techniques of overlapping have weakly improved the execution time. On the TN310, the results show that good improvements are obtained, but the communications are not completely overlapped. This corroborate with the theoretical study. Note that the estimated machine parameters, for the TN310, under PVM environment, are $\beta=0.002 s, \tau=22 \mu \mathrm{s}$ and $\omega=0.25 \mu \mathrm{s}$.

Table 1. Execution times in seconds for one iteration on the cluster

\begin{tabular}{|c||c|c||c|c||c|c||c|c||c|c||c|c|}
\hline \multicolumn{1}{|l|}{$\mathrm{n}$} & \multicolumn{2}{|c||}{128} & \multicolumn{2}{c||}{256} & \multicolumn{2}{c||}{512} & \multicolumn{2}{c||}{768} & \multicolumn{2}{c||}{1024} & \multicolumn{2}{c|}{2048} \\
\hline $\mathrm{p}$ & n_ov & str1 & n_ov & str1 & n_ov & str1 & n_ov & str1 & n_Ov & str1 & n_ov & str1 \\
\hline 2 & 0.06 & 0.05 & 0.64 & 0.66 & 6.62 & 5.81 & 20.32 & 19.81 & 48.57 & 47.07 & 391.51 & 377.25 \\
\hline 4 & 0.04 & 0.04 & 0.25 & 0.26 & 3.08 & 2.97 & 10.39 & 10.1 & 24.11 & 23.62 & 191.81 & 190.51 \\
\hline 8 & 0.03 & 0.03 & 0.16 & 0.16 & 1.56 & 1.37 & 5.65 & 5.25 & 13.11 & 12.31 & 102.45 & 97.52 \\
\hline 16 & 0.04 & 0.04 & 0.13 & 0.12 & 0.68 & 0.69 & 2.9 & 2.72 & 7.14 & 6.66 & 53.7 & 51.56 \\
\hline 32 & 0.03 & 0.03 & 0.12 & 0.11 & 0.49 & 0.46 & 1.39 & 1.4 & 3.61 & 3.51 & 28.83 & 27.3 \\
\hline
\end{tabular}

Table 2. Execution times in seconds for one iteration on the TN310

\begin{tabular}{|c||c|c||c|c||c|c||c|c||c|c|}
\hline \multicolumn{1}{|c||}{$\mathrm{n}$} & \multicolumn{2}{|c||}{128} & \multicolumn{2}{c||}{256} & \multicolumn{2}{c||}{512} & \multicolumn{2}{c||}{768} & \multicolumn{2}{c|}{1024} \\
\hline $\mathrm{p}$ & n_Ov & str1 & n_ov & str1 & n_Ov & str1 & n_ov & str1 & n_ov & str1 \\
\hline 2 & 14.42 & 10.35 & 109.05 & 72.32 & 941.17 & 672.46 & & & & \\
\hline 4 & 6.65 & 5.47 & 45.42 & 36.66 & 471.77 & 374.81 & 1562.94 & 1267.31 & & \\
\hline 8 & 3.94 & 3.55 & 23.53 & 20.38 & 172.34 & 140.71 & 791.51 & 472.02 & 1857.85 & 1576.82 \\
\hline 16 & 3.9 & 2.8 & 13.58 & 12.52 & 89.76 & 77.03 & 286.9 & 249.29 & 669.22 & 576.8 \\
\hline
\end{tabular}

The table 3, shows that the lower execution time, on 16 processors, for the second strategie, is obtained for $q=1$. This means that it is not necessary to subdivide the columns to be translated. This result corroborates with the theoretical study. Note that the algorithm for the case $q=1$ is not identical to the algorithm proposed for the first strategie. 
Table 3. Execution times on the TN310, with pipeline for 16 processors, using different values of $q$

\begin{tabular}{|c|c|c|c|c|c|}
\hline & \multicolumn{5}{|c|}{$\mathrm{n}$} \\
\hline $\mathrm{q}$ & 128 & 256 & 512 & 768 & 1024 \\
\hline 1 & 2.7 & 11.49 & 74.57 & 236.48 & 554.17 \\
\hline 2 & 3.23 & 12.94 & 76.94 & 240.21 & 554.82 \\
\hline 4 & 4.33 & 14.91 & 81.96 & 252.3 & 564.41 \\
\hline 8 & 6.43 & 19.43 & 89.81 & 264.92 & 572.7 \\
\hline
\end{tabular}

\section{Conclusion}

In this paper, we have proposed two algorithms, which enable the overlapping of the communications by the computations in the parallelization of the one-sided Jacobi method. Theses techniques are experimented under MPI on a cluster of PCs and under PVM on the parallel system TN310, since MPI is not implemented on our system.

The experimental execution times obtained on the TN310 corroborate with the theoretical results. The results obtained on the cluster are not those expected. We think that the overlap is not available with this platform. The proposed algorithms can be easily extended to the block one-sided Jacobi version.

Acknowledgment. The authors thanks the anonymous reviewers for there valuable comments.

\section{References}

1. http://icluster.imag.fr, "The icluster project".

2. J. Cuenca, D. Giménez, "Implementation of parallel one-sided block Jacobi methods for the symmetric eigenvalue problem", ParCo'99, Imperial College Press (2000), 291-298.

3. G.H. Golub, C.F. Van Loan, "Matrix computation", Johns Hopkins University Press, 1989, 2nd edition.

4. E.M. Daoudi, A. Lakhouaja, "Exploiting the symmetry in the parallelization of the Jacobi method", Parallel Computing 23 (1997), 137-151.

5. E.M. Daoudi, A. Lakhouaja, H. Outada, "Study of the parallel block one-sided Jacobi method", HPCN'2001, LNCS 2110 Springer Velag, 2001, 454-463.

6. P.J. Eberlein, H. Park, "Efficient implementation of Jacobi algorithms and Jacobi sets on distributed memory architectures", Journal of Parallel and Distributed Computing 8 (1990), 358-366.

7. F.T. Luck, H. Park, "On parallel Jacobi orderings", SIAM J. Sci Stat. Comp. 10 (1989), 18-26.

8. M. Pourzandi, B. Tourancheau, "A parallel performance study of Jacobi-like eigenvalue solution", In Proceeding of First International Meeting on Vector and Parallel Processing, Porto, Portugal, September 1993. 